\title{
Résumé des recommandations du Comité consultatif de la médecine tropicale et de la médecine des voyages (CCMTMV) sur les problèmes liés au paludisme chez certains hôtes
}

\section{Boggild A. ${ }^{1}$, Brophy J. ${ }^{2}$, Charlebois P. ${ }^{3}$, Crockett M. ${ }^{4}$, Geduld J. ${ }^{5}$, Ghesquiere W.6, McDonald P.7, Plourde P. ${ }^{8}$, Teitelbaum P. ${ }^{9}$, Tepper M. ${ }^{10}$, Schofield S. ${ }^{11}$, McCarthy A. (présidente) ${ }^{12}$}

${ }^{1}$ University Health Network, Toronto General Hospital (Toronto, Ont.)

${ }^{2}$ Division des maladies infectieuses, Centre hospitalier pour enfants de l'est de l'Ontario (Ottawa, Ont.)

${ }^{3}$ Médecine interne, Centre des services de santé des Forces canadiennes (Atlantique) (Halifax, N.-É.)

${ }^{4}$ Pédiatrie et santé des enfants, Université du Manitoba (Winnipeg, Man.)

${ }^{5}$ Direction générale de la prévention et du contrôle des maladies infectieuses, Agence de la santé publique du Canada (Ottawa, Ont.)

${ }^{6}$ Infectious Diseases and Internal Medicine, Université de la Colombie-Britannique (Victoria, C.-B.)

${ }^{7}$ Direction des produits thérapeutiques, Santé Canada (Ottawa, Ont.)

${ }^{8}$ Faculté de médecine, Université du Manitoba (Winnipeg, Man.)

${ }^{9}$ Clinique santé-voyage Riverside (Ottawa, Ont.)

${ }^{10}$ Programme de lutte contre les maladies transmissibles, Direction de la protection de la santé de la Force (Ottawa, Ont.)

${ }^{11}$ Entomologie de la lutte antiparasitaire, Direction de la protection de la santé de la Force (Ottawa, Ont.)

${ }^{12}$ Clinique de médecine tropicale et de santé internationale, Division des maladies infectieuses, Campus général de

l'Hôpital d'Ottawa (Ottawa, Ont.)

*Auteure-ressource : AMcCARTHY@Ottawahospital.on.ca

\section{Résumé}

Contexte : Au nom de l'Agence de la santé publique du Canada, le Comité consultatif de la médecine tropicale et de la médecine des voyages (CCMTMV) a formulé les Recommandations canadiennes pour la prévention et le traitement du paludisme (malaria) chez les voyageurs internationaux à l'intention des fournisseurs de soins de santé qui préparent les patients qui voyageront dans des régions impaludées et qui traitent les voyageurs qui sont revenus malades au pays.

Objectif : Donner des lignes directrices sur les problèmes liés au paludisme touchant certains hôtes.

Méthodes : Le CCMTMV a examiné toutes les sources principales de renseignements sur la prévention du paludisme, de même que les recherches récentes et les données épidémiologiques nationales et internationales afin de proposer des lignes directrices adaptées au contexte canadien. Il a formulé ses recommandations dans le cadre d'une approche de la médecine fondée sur les preuves à l'aide d'échelles d'évaluation afin de déterminer le poids et la qualité des preuves.

Recommandations : Toutes les personnes visitant des régions impaludées devraient prendre des mesures de protection individuelle efficaces (insectifuges topiques, moustiquaires de lit, choix de comportement) et observer la chimioprophylaxie prescrite. La chimioprophylaxie pour les femmes enceintes et les femmes qui allaitent, et les enfants, requiert une attention particulière dans le contexte du trimestre de grossesse, de l'âge ou de la taille du nourrisson/de l'enfant et de l'état de la glucose-6-phosphate déshydrogénase (G-6-PD). Les recommandations pour les voyageurs faisant un séjour de longue durée, les expatriés et les personnes visitant des amis et de la famille ne sont pas sensiblement différentes de celles pour les voyageurs à court terme. Certains problèmes médicaux sousjacents peuvent rendre les personnes plus vulnérables au paludisme. En outre, certaines affections ou leur traitement peuvent empêcher l'usage d'un ou de plusieurs antipaludéens. 


\section{Introduction}

Le paludisme est une maladie infectieuse grave causée par cinq différentes espèces de Plasmodium : P. falciparum, $P$. vivax, $P$. ovale, $P$. malariæ et $P$. knowlesi. Le paludisme est transmis par la morsure d'un moustique anophèle femelle infecté. Les infections à P. falciparum sont associées aux taux de mortalité les plus élevés. Le taux de mortalité global du paludisme à $P$. falciparum varie d'environ $1 \%$ à $5 \%$ et grimpe pour atteindre $20 \%$ dans les cas graves(1).

Le Comité consultatif de la médecine tropicale et de la médecine des voyages (CCMTMV) donne de façon continue à l'Agence de la santé publique du Canada des conseils opportuns de nature médicale, scientifique et de santé publique concernant les maladies tropicales et les risques pour la santé associés aux voyages internationaux. Il s'agit d'un résumé d'une section des Recommandations canadiennes pour la prévention et le traitement du paludisme (malaria) chez les voyageurs internationaux, du CCMTMV, formulées à l'intention des fournisseurs de soins de santé qui préparent les patients qui voyageront dans des régions impaludées et qui traitent les voyageurs qui sont revenus malades au pays(2). Ces lignes directrices comprennent une description complète des recommandations sur l'évaluation des risques, la prévention et le traitement du paludisme, une maladie qui demeure encore peu connue au Canada. Deux résumés supplémentaires des lignes directrices sont disponibles, ils sont axés sur la prévention et le traitement du paludisme $(3,4)$.

Certains groupes de voyageurs sont exposés à différents risques d'infection palustre par rapport au voyageur moyen. S'ils ne peuvent pas reporter leur voyage dans des régions à risque élevé de paludisme, les femmes enceintes et les femmes qui allaitent, et les jeunes enfants doivent recevoir une chimioprophylaxie antipaludéenne adaptée, car certains médicaments sont contre-indiqués. Les voyageurs faisant un séjour de longue durée, les expatriés et les personnes visitant des amis et de la famille peuvent percevoir différemment le risque de paludisme et observer différemment la chimioprophylaxie. Ils ont besoin d'obtenir de plus amples renseignements sur l'autodiagnostic et le traitement. Le problème des médicaments de contrefaçon est traité de façon particulière pour les voyageurs faisant un séjour de longue durée.

\section{Méthodes}

Le sous-comité sur le paludisme, un groupe de travail du CCMTMV, a élaboré les lignes directrices. Le processus mis en place pour les concevoir a été décrit précédemment(3). Il comprenait un examen des recherches récentes et des données épidémiologiques nationales et internationales ainsi que la prise en compte d'autres facteurs, tels que l'épidémiologie du paludisme, ainsi que les valeurs et les préférences des voyageurs et des fournisseurs de soins de santé. Les recommandations dans le cadre d'une approche de la médecine fondée sur les preuves pour divers problèmes liés au paludisme touchant certains hôtes ont été formulées à l'aide d'échelles d'évaluation afin de déterminer le poids et la qualité des preuves.

\section{Recommandations}

Les recommandations du CCMTMV dans le cadre d'une approche de la médecine fondée sur les preuves en ce qui concerne la prévention et le traitement du paludisme chez certains hôtes sont résumées dans le Tableau 1. Une analyse de certaines recommandations principales suivra.

\section{Enfants}

Le paludisme touche de façon disproportionnée les enfants et peut se manifester par des symptômes qui ne sont pas spécifiques et qui ressemblent à d'autres maladies courantes chez l'enfant, ce qui peut retarder le diagnostic. Un paludisme grave ou avec complications, comme l'accès pernicieux à forme cérébrale, une anémie grave, un choc ou même la mort, peut se développer plus rapidement chez les enfants(5).

Il faut éviter aux jeunes enfants les voyages dans des régions de transmission intense du paludisme, surtout si la maladie est résistante à la chloroquine(6). Lorsque les voyages dans des régions impaludées sont inévitables :

- Tous les enfants devraient prendre des mesures de protection individuelle efficaces (insectifuges topiques, moustiquaires de lit, choix de comportement) (7) et observer une chimioprophylaxie antipaludéenne appropriée(1,8). 
- Pour les régions où la maladie est résistante à la chloroquine, la méfloquine, la doxycycline ( $\geq 8$ ans) et l'atovaquone/proguanil ( $\geq 5 \mathrm{~kg}$ ) sont les médicaments les plus appropriés (9-12).

- La primaquine peut convenir aux enfants qui ne peuvent prendre des agents prophylactiques de première intention, une fois que le taux de G-6-PD a été confirmé(13).

- Prescrire des antipaludéens pour les bébés allaités même si leur mère prend des antipaludéens $(6,14)$

- Instructions précises relatives au dosage :

- Prescrire suffisamment de comprimés, car il se pourrait que quelques doses soient vomies ou recrachées. Donner des instructions claires quant au moment où les doses que le patient n'a pas réussi à ingérer doivent être répétées.

- Demander de couper au préalable ou d'écraser les comprimés, et les insérer dans des capsules pour un dosage plus précis et facile.

- Décrire comment ajuster la dose des médicaments afin de l'augmenter à mesure que le poids de l'enfant augmente.

- Expliquer qu'il existe peu de préparations pédiatriques et que pour cette raison, les comprimés antipaludéens peuvent être écrasés et mélangés avec quelque chose de sucré pour cacher leur goût désagréable.

\section{Femmes enceintes et femmes qui allaitent}

Les femmes enceintes devraient autant que possible reporter leur voyage dans des régions impaludées, surtout dans les régions où elles risquent de contracter le paludisme à $P$. falciparum pharmacorésistant(15). Le paludisme accroît le risque de décès chez la femme enceinte et le nouveau-né, ainsi que le risque de fausse couche et de mortinaissance. Les femmes qui suivent une prophylaxie inefficace sont proportionnellement plus nombreuses à accoucher d'un bébé de faible poids(15).

Si le voyage est inévitable :

- les femmes enceintes et les femmes qui allaitent devraient prendre des mesures de protection individuelle (insectifuges topiques, moustiquaires de lit imprégnées d'insecticide, choix de comportement) (17);

- Prescrire la chimioprophylaxie selon la destination :

- de la chloroquine dans les régions où les souches sont sensibles à la chloroquine;

- de la méfloquine là où l'exposition au paludisme à $P$. falciparum résistant à la chloroquine est inévitable (17-19);

- discuter des avantages et des risques associés à l'atovaquone/proguanil après le premier trimestre chez les femmes qui ne peuvent éviter de voyager dans des zones où le paludisme est résistant à la méfloquine ou qui ne peuvent pas prendre la méfloquine(20,21);

○ bien qu'ils puissent être pris sans danger durant la grossesse, la chloroquine et le proguanil sont inadéquats en tant qu'antipaludéens et ne peuvent être recommandés dans les zones de chloroquinorésistance(22);

○ La doxycycline est contre-indiquée pendant la grossesse.

Les femmes qui allaitent devraient continuer de le faire si elles ont recours à une chimioprophylaxie qui est sûre pour les nourrissons (chloroquine, méfloquine, atovaquone/proguanil chez les nourrissons de $\geq 5 \mathrm{~kg}$ ). L'absorption de la doxycycline par le lait maternel est probablement négligeable et l'allaitement n'est pas une contre-indication absolue de l'usage de la doxycycline par la mère(23).

\section{Migrants :}

Bien que la plupart des cas se développent dans les trois mois suivant la dernière exposition, le paludisme pourrait être la raison d'une fièvre qui se développe dans les 12 mois suivant le départ de la région impaludée(24). Le risque de paludisme existe pour les migrants après leur arrivée au Canada.

- Test de dépistage du paludisme chez les migrants ayant une fièvre inexpliquée depuis au moins 12 mois, après leur arrivée au Canada; 
- Envisager un dépistage du paludisme chez les migrants asymptomatiques nouvellement arrivés et qui proviennent de zones endémiques élevées, et traiter les cas qui présentent une parasitémie (hormis la présence de gamétocytes seulement) dans les frottis sanguins;

- Demander aux migrants provenant de pays impaludés quels sont leurs projets de voyage afin de leur fournir des conseils sur le paludisme par anticipation(25).

Voyageurs faisant un séjour de longue durée, expatriés et personnes visitant des amis et de la famille

Les recommandations pour la prévention du paludisme chez les voyageurs faisant un séjour de longue durée (plus d'un mois), les expatriés et les personnes visitant des amis et de la famille, sont très semblables aux recommandations standard pour les voyageurs faisant un bref séjour(26): utiliser la chimioprophylaxie antipaludéenne prescrite et les mesures de protection individuelle systématiquement, y compris les moustiquaires de lit imprégnées d'insecticide et les insectifuges topiques contenant 20 à $30 \%$ de DEET ou $20 \%$ d'icaridine.

Voici certains sujets à aborder lorsque l'on conseille des expatriés et des voyageurs faisant un long séjour à propos de la prévention du paludisme :

- préoccupation possible concernant l'innocuité avec une utilisation prolongée de médicaments chimioprophylactiques;

- recours aux mesures de protection individuelle à long terme;

- coût des médicaments à long terme;

- utilisation de médicaments achetés localement qui pourraient être contrefaits;

- conseils contradictoires concernant la chimioprophylaxie et l'auto-traitement;

- nécessité d'observance continue de la chimioprophylaxie.

Étant donné que le taux général de non-observance de la chimioprophylaxie est aussi élevé que 61 \%(27), les conseils offerts avant le départ devraient être axés sur les éléments suivants :

- les symptômes et le risque du paludisme, en mettant l'accent sur la nécessité d'un diagnostic et d'un traitement précoces;

- l'élaboration d'un plan d'accès à des soins médicaux satisfaisants en cas de maladie;

- un traitement de réserve d'urgence (auto-traitement);

- l'abordabilité de la chimioprophylaxie;

- la probabilité que les médicaments antipaludéens locaux soient contrefaits(28);

- la perte de l'immunité partielle chez les personnes visitant des amis et la famille en raison de la résidence dans un pays sans paludisme(25).

Le risque de paludisme chez les personnes visitant des amis et la famille est presque le même que celui pour les résidents locaux. Par contre, le risque de maladie grave est plus élevé en raison de la perte d'immunité partielle après avoir vécu dans une région non endémique(25).

Les personnes visitant des amis et la famille ont tendance à :

- être moins susceptibles de rechercher des conseils sanitaires préventifs à l'intention des voyageurs ou de s'y conformer (29-31), probablement en raison de :

- contraintes financières ou de temps(25);

- fausses idées sur le risque de maladie et l'immunité;

- la confiance accordée aux conseils de membres de la famille ou de fournisseurs locaux à leur destination $(25-27,32,33)$.

- rester dans des endroits ruraux (présentant des taux plus élevés de transmission du paludisme que les centres urbains) pendant plus longtemps(25);

- $\quad$ séjourner chez des membres de la famille locale plutôt que dans des hôtels climatisés et munis de bonnes moustiquaires(25);

- voyager avec leurs enfants nés au Canada(25); 
- faire des plans de voyage d'urgence à la dernière minute(25).

Tableau 1 : Recommandations dans le cadre d'une approche de la médecine fondée sur les preuves en ce qui concerne la prévention et le traitement du paludisme chez certains hôtes

\begin{tabular}{|c|c|c|}
\hline \multicolumn{2}{|c|}{ Recommandation } & $\begin{array}{l}\text { Catégorie } \\
\text { MFP1 }\end{array}$ \\
\hline \multicolumn{3}{|c|}{ Enfants } \\
\hline 1. & $\begin{array}{l}\text { Il faut éviter aux jeunes enfants les voyages dans des régions de transmission intense du paludisme, } \\
\text { surtout si la maladie est résistante à la chloroquine(6). }\end{array}$ & C III \\
\hline 2. & $\begin{array}{l}\text { Tous les enfants qui se rendent dans des régions impaludées devraient prendre des mesures de } \\
\text { protection individuelle(7). }\end{array}$ & A I \\
\hline 3. & $\begin{array}{l}\text { Dans des zones de chloroquinorésistance, la méfloquine, la doxycycline ( } \geq 8 \text { ans) et } \\
\text { l'atovaquone/proguanil }(\geq 5 \mathrm{~kg} \text { ) sont les médicaments de choix pour la chimioprophylaxie(9-12). }\end{array}$ & A I \\
\hline 4. & $\begin{array}{l}\text { La chimioprophylaxie à la primaquine peut convenir aux enfants qui ne peuvent prendre aucun des } \\
\text { agents prophylactiques de première intention, après confirmation du statut de G-6-PD(13). }\end{array}$ & B II \\
\hline \multicolumn{3}{|c|}{ Femmes enceintes } \\
\hline 5. & $\begin{array}{l}\text { Les femmes enceintes devraient éviter de voyager dans des régions de transmission intense du } \\
\text { paludisme(15). }\end{array}$ & C III \\
\hline 6. & $\begin{array}{l}\text { Les femmes enceintes qui voyagent dans des régions impaludées devraient prendre des mesures de } \\
\text { protection individuelle, notamment utiliser des insectifuges topiques appropriés et des } \\
\text { moustiquaires de lit imprégnées d'insecticide(16). }\end{array}$ & A I \\
\hline 7. & $\begin{array}{l}\text { Dans les régions où le paludisme est sensible à la chloroquine, les femmes enceintes devraient } \\
\text { prendre de la chloroquine à titre prophylactique. }\end{array}$ & A I \\
\hline 8. & $\begin{array}{l}\text { Lorsqu'on ne peut éviter l'exposition au paludisme à P. falciparum résistant à la chloroquine, les } \\
\text { femmes enceintes devraient prendre de la méfloquine de la conception au premier trimestre (A II) de } \\
\text { même que durant les deuxième et troisième trimestres de grossesse (A I)(17-19). }\end{array}$ & A II, A I \\
\hline 9. & $\begin{array}{l}\text { Il n'existe actuellement aucun antipaludéen approuvé chez les femmes enceintes qui voyagent dans } \\
\text { des régions où le paludisme est résistant à la méfloquine. L'administration d'atovaquone/proguanil } \\
\text { peut être envisagée après le premier trimestre après avoir bien soupesé les avantages et les } \\
\text { risques }(20,21) \text {. }\end{array}$ & B II \\
\hline 10. & $\begin{array}{l}\text { Bien qu'ils puissent être pris sans danger durant la grossesse, la chloroquine et le proguanil sont } \\
\text { inadéquats en tant qu'antipaludéens et ne peuvent être recommandés dans les zones de } \\
\text { chloroquinorésistance(22). }\end{array}$ & E I \\
\hline \multicolumn{3}{|c|}{ Femmes qui allaitent } \\
\hline 11. & $\begin{array}{l}\text { Les nourrissons devraient recevoir leur propre traitement chimioprophylactique adapté, même s'ils } \\
\text { sont allaités(23). }\end{array}$ & A III \\
\hline 12. & Les femmes qui allaitent un enfant de moins de 5 kg devraient éviter l'atovaquone/proguanil(23). & C II \\
\hline 13. & $\begin{array}{l}\text { Des données limitées semblent indiquer que l'absorption de la doxycycline par le lait maternel est } \\
\text { négligeable et que l'allaitement n'est pas une contre-indication absolue de l'usage de la doxycycline } \\
\text { par la mère(23). }\end{array}$ & C III \\
\hline \multicolumn{3}{|c|}{ Migrants } \\
\hline 14. & $\begin{array}{l}\text { Test de dépistage du paludisme chez les migrants ayant une fièvre inexpliquée depuis au moins } \\
12 \text { mois, après leur arrivée au Canada. }\end{array}$ & C III \\
\hline 15. & $\begin{array}{l}\text { Envisager un dépistage du paludisme chez les migrants asymptomatiques nouvellement arrivés et } \\
\text { qui proviennent de zones endémiques élevées, et traiter les cas qui présentent une parasitémie } \\
\text { (hormis la présence de gamétocytes seulement) dans les frottis sanguins. }\end{array}$ & C III \\
\hline 16. & $\begin{array}{l}\text { Demander aux migrants provenant de pays impaludés quels sont leurs projets de voyage. Cela peut } \\
\text { donner l'occasion d'offrir des conseils de prévention concernant le paludisme(25). }\end{array}$ & C III \\
\hline \multicolumn{3}{|c|}{ Voyageurs faisant un séjour de longue durée ou expatriés } \\
\hline 17. & $\begin{array}{l}\text { Les recommandations relatives à la prévention du paludisme chez les personnes qui voyagent ou qui } \\
\text { séjournent longtemps à l'étranger ne devraient pas différer sensiblement de celles qui s'appliquent } \\
\text { aux courts séjours(26). }\end{array}$ & B III \\
\hline 18. & $\begin{array}{l}\text { Il est raisonnable d'apprendre aux voyageurs qui partent pour une longue période à se servir des } \\
\text { tests de diagnostic rapide }(26,34) \text {. }\end{array}$ & C III \\
\hline 19. & $\begin{array}{l}\text { Informer les voyageurs qui séjourneront longtemps à l'étranger et qui risquent d'acheter des } \\
\text { médicaments dans des pays où il n'y a pas de contrôle de qualité au sujet des antipaludéens de } \\
\text { contrefaçon (35-37). }\end{array}$ & C II \\
\hline 20. & La primaquine devrait être considérée comme le médicament de choix pour la prophylaxie finale & A I \\
\hline
\end{tabular}




\begin{tabular}{|c|c|c|}
\hline & $\begin{array}{l}\text { chez les militaires, les voyageurs partis pour une longue période ou les expatriés qui reviennent de } \\
\text { régions de transmission de } P \text {. vivax }(26,38,39) \text {. }\end{array}$ & \\
\hline \multicolumn{3}{|c|}{ Personnes visitant des amis et la famille } \\
\hline 21. & $\begin{array}{l}\text { Aviser les Canadiens qui rendent visite à des amis et parents et qui voyagent dans des pays } \\
\text { impaludés du risque de paludisme, y compris la perte de l'immunité partielle liée au fait de vivre au } \\
\text { Canada et du risque accru de maladie grave chez les enfants et les femmes enceintes(25). }\end{array}$ & C III \\
\hline 22. & $\begin{array}{l}\text { Prodiguer des conseils aux Canadiens qui rendent visite à des amis et parents et qui voyagent dans } \\
\text { des pays impaludés concernant les mesures de protection individuelle (répulsifs, moustiquaires de } \\
\text { lit, choix de comportement) et la chimioprophylaxie(25). }\end{array}$ & C III \\
\hline 23. & $\begin{array}{l}\text { Discuter de l'abordabilité de la chimioprophylaxie pour les Canadiens qui rendent visite à des amis et } \\
\text { parents et qui voyagent dans des pays impaludés, en tenant compte du coût au moment de faire des } \\
\text { choix(25). }\end{array}$ & C III \\
\hline \multicolumn{3}{|c|}{ Voyageurs souffrant de comorbidités } \\
\hline 24. & $\begin{array}{l}\text { Les personnes qui sont immunodéprimées ou souffrent de maladies concomitantes devraient } \\
\text { consulter un spécialiste en médecine des voyages ou en maladies infectieuses(40). }\end{array}$ & B III \\
\hline 25. & $\begin{array}{l}\text { Avant de prescrire des antipaludéens à des personnes atteintes de problèmes de santé chroniques, y } \\
\text { compris les personnes infectées par le VIH, il convient d'examiner soigneusement les interactions } \\
\text { médicamenteuses potentielles et les chevauchements de toxicités(41). }\end{array}$ & A I \\
\hline 26. & $\begin{array}{l}\text { Les personnes infectées par le VIH qui sont enceintes ou qui souffrent d'une immunosuppression } \\
\text { avancée doivent être incitées à choisir des régions où le paludisme n'est pas endémique ou à différer } \\
\text { leur voyage jusqu'à la fin de leur grossesse ou jusqu'à la restauration de leur fonction immunitaire. }\end{array}$ & B III \\
\hline 27. & $\begin{array}{l}\text { Offrir un traitement de réserve antipaludéen aux voyageurs aspléniques qui peuvent subir des } \\
\text { retards lorsqu'il s'agit d'avoir accès à des soins appropriés en cas de maladie fébrile. }\end{array}$ & A II \\
\hline 28. & $\begin{array}{l}\text { Avant qu'une personne traitée à la warfarine prenne de la méfloquine, de la doxycycline ou du } \\
\text { proguanil (y compris dans l'association atovaquone/proguanil), un essai préalable avec dosage du } \\
\text { RIN devrait être effectué (42-45). }\end{array}$ & A II \\
\hline 29. & Éviter la chloroquine et la méfloquine en présence de troubles épileptiques chroniques. & E II \\
\hline 30. & Éviter la chloroquine et la méfloquine chez les voyageurs souffrant de myasthénie grave. & E III \\
\hline 31. & $\begin{array}{l}\text { Passer soigneusement en revue les antécédents en matière de santé mentale avant de prescrire de la } \\
\text { méfloquine pour s'assurer de l'absence de troubles psychotiques, dépressifs ou anxieux(46). }\end{array}$ & A I \\
\hline 32. & $\begin{array}{l}\text { La chloroquine peut exacerber le psoriasis. La méfloquine, la doxycycline et l'association } \\
\text { atovaquone/proguanil sont préférables à la chloroquine chez les patients souffrant d'un psoriasis } \\
\text { sous-jacent. }\end{array}$ & B III \\
\hline 33. & $\begin{array}{l}\text { La primaquine ne doit pas être utilisée en tant que chimioprophylaxie en présence de déficit en G-6- } \\
\text { PD. }\end{array}$ & E II \\
\hline 34. & $\begin{array}{l}\text { L'association atovaquone/proguanil peut être le premier choix en tant que prophylaxie } \\
\text { antipaludéenne en présence de porphyrie. }\end{array}$ & B III \\
\hline
\end{tabular}

$1 \quad$ MFP = Médecine fondée sur les preuves Les catégories MFP sont les suivantes :

Force de la recommandation

A =Données suffisantes pour recommander l'utilisation

$\mathrm{B}=$ Données acceptables pour recommander l'utilisation

$\mathrm{C}=$ Données insuffisantes pour recommander ou déconseiller l'utilisation

$\mathrm{D}=$ Données acceptables pour déconseiller l'utilisation

$\mathrm{E}=$ Données suffisantes pour déconseiller l'utilisation

Qualité des preuves

I= Données obtenues dans le cadre d'au moins un essai comparatif convenablement randomisé

II=Données obtenues dans le cadre d'au moins un essai clinique bien conçu, sans randomisation, d'études de cohortes ou d'études analytiques cas-témoins, réalisés de préférence dans plus d'un centre, à partir de plusieurs séries chronologiques, ou résultats spectaculaires d'expériences non comparatives.

III=Opinions exprimées par des sommités dans le domaine et reposant sur l'expérience clinique, des études descriptives ou des rapports de comités d'experts.

\section{Innocuité à long terme de la chimioprophylaxie et mesures de protection individuelle}

L'utilisation à long terme de la chimioprophylaxie recommandée pour les courts séjours à l'étranger n'entraîne pas un risque additionnel d'événements indésirables graves bien que les données sur l'efficacité et la tolérance des régimes 
recommandés soient limitées. Le Tableau 2 résume l'innocuité de la chimioprophylaxie pour l'utilisation à long terme.

Tableau 2 : Innocuité de la chimioprophylaxie pour l'utilisation à long terme

\begin{tabular}{|l|l|}
\hline $\begin{array}{l}\text { Médicament } \\
\text { chimioprophylactique }\end{array}$ & Effets de l'utilisation à long terme \\
\hline Chloroquine & $\begin{array}{l}\text { Exige un examen ophtalmologique au moins tous les deux ans (31). Cependant, la } \\
\text { chloroquine est rarement indiquée, car la résistance à la chloroquine est très } \\
\text { répandue. }\end{array}$ \\
\hline Méfloquine & $\begin{array}{l}\text { Bien tolérée (47-50). } \\
\text { La tolérance à la méfloquine s'améliore avec le temps, peut-être parce que les } \\
\text { événements indésirables se manifestent relativement tôt (47). Par conséquent, il ne } \\
\text { semble pas qu'une utilisation prolongée augmente les risques (28). }\end{array}$ \\
\hline Atovaquone/proguanil & $\begin{array}{l}\text { Bien que les données sur l'utilisation prolongée de l'atovaquone/proguanil soient } \\
\text { limitées, les composants individuels ont été utilisés pendant de longues périodes } \\
\text { (30). }\end{array}$ \\
\hline Doxycycline & $\begin{array}{l}\text { Bien que les données soient limitées, il convient de signaler que ce médicament et la } \\
\text { minocycline associée ont été utilisés pendant de longues périodes pour d'autres } \\
\text { indications (31). }\end{array}$ \\
\hline
\end{tabular}

À l'heure actuelle, il n'y aucun enregistrement de moustiquaires imprégnées d'insecticide à longue durée d'action au Canada. Les moustiquaires imprégnées d'insecticide peuvent être obtenues auprès de certaines cliniques santévoyage canadiennes et d'autres fournisseurs nationaux et internationaux (8);

- L'insecticide imprégné dans la plupart des moustiquaires de lit commence à perdre son effet après 6 mois (8);

- La perméthrine liquide utilisée pour traiter les moustiquaires de lit n'est pas vendue au Canada;

- Les voyageurs devraient renouveler le traitement à l'insecticide de leurs moustiquaires de lit au début de la saison des pluies.

\section{Médicaments de contrefaçon}

De nombreux expatriés et voyageurs faisant de longs séjours peuvent avoir l'occasion d'acheter leurs médicaments chimioprophylactiques et antipaludéens sans ordonnance dans les pharmacies locales, et ne sont pas en mesure d'évaluer l'authenticité de ces médicaments.

Il convient d'encourager tous les voyageurs et expatriés à acheter une provision de médicaments dans des pays qui ont mis en place des mesures strictes de contrôle de la qualité (35-37).

Pour l'achat à l'extérieur du Canada :

- Le Coartem ${ }^{\circledR}$ (artéméther/luméfantrine) n'est pas encore homologué au Canada, mais est recommandé par l'OMS comme traitement de première intention contre le paludisme à P. falciparum. Les voyageurs devraient acheter ce médicament en Europe, aux États-Unis ou dans d'autres pays où une contrefaçon est peu probable (39).

- La prophylaxie par l'atovaquone/proguanil peut être trop coûteuse pour la majorité des utilisations à long terme. Les voyageurs faisant de longs séjours et les expatriés peuvent choisir d'acheter assez de médicaments pour une ou deux cures d'auto-traitement (51).

\section{Tests diagnostiques rapides}

Les tests diagnostiques rapides sont des outils diagnostiques essentiels lorsque l'on ne peut obtenir les résultats de la microscopie pour le paludisme en moins de 2 heures (26). Les tests diagnostiques rapides sont simples à utiliser et n'exigent pas de matériel de laboratoire spécialisé ni de compétences particulières et peuvent être des accessoires très utiles pour le diagnostic du paludisme (52). Toutefois, bon nombre de voyageurs sont incapables d'effectuer les procédures ou d'interpréter correctement les résultats $(26,53,54)$. Sans la formation adéquate du personnel de 
laboratoire, l'utilité des tests diagnostiques rapides peut ne pas être meilleure parmi les expatriés $(34,55)$.

Néanmoins, les membres clés d'une communauté raisonnable d'expatriés pourraient être formés à l'utilisation et à l'administration d'un auto-traitement approprié $(26,34)$.

\section{Auto-traitement de réserve d'urgence}

L'auto-traitement est une mesure salvatrice temporaire à prendre dans un délai de 24 heures pendant qu'on tente d'obtenir l'aide d'un médecin. Les personnes qui se rendent dans une région présentant un risque élevé ne devraient pas compter uniquement sur l'auto-traitement (40,56-58). Les régimes d'auto-traitement par région sont résumés dans le Tableau 3.

Les raisons de l'auto-traitement comprennent le voyage et le séjour :

- en Afrique subsaharienne où surviennent $90 \%$ de tous les cas de morbidité et de mortalité associés au paludisme;

- dans les régions isolées où l'accès aux soins de santé est un problème;

- dans les régions où le risque de paludisme est faible et où l'auto-traitement est préférable à la prophylaxie à long terme $(26,28,56,59)$.

Le traitement de réserve par l'atovaquone/proguanil ou la quinine et la doxycycline est recommandé pour les voyageurs qui ne peuvent obtenir un diagnostic dans les 24 heures.

Tableau 3 : Régime d'auto-traitement

\begin{tabular}{|l|l|}
\hline Région & Régime d'auto-traitement \\
\hline $\begin{array}{l}\text { Régions où les souches sont sensibles à la } \\
\text { chloroquine }\end{array}$ & $\begin{array}{l}\text { Il convient d'amorcer l'auto-traitement à la chloroquine, puis } \\
\text { continuer ou amorcer une prophylaxie à la chloroquine }(54,56,60) .\end{array}$ \\
\hline $\begin{array}{l}\text { Régions où la souche } \text { P. falciparum est } \\
\text { résistante à la chloroquine ou à la } \\
\text { chloroquine et à la méfloquine }\end{array}$ & $\begin{array}{l}\text { Il convient d'amorcer l'auto-traitement avec un médicament } \\
\text { différent de celui utilisé pour la prophylaxie : }\end{array}$ \\
& $\begin{array}{l}\left.\text { a. atovaquone/proguanil (Malarone }{ }^{\circledR}\right) \text { ou } \\
\text { b. quinine et doxycycline par voie orale ou } \\
\text { c. artéméther/luméfantrine (Coartem }{ }^{\circledR} \text { ), acheté dans un pays où les } \\
\text { normes de contrôle de la qualité sont rigoureuses afin de réduire au } \\
\text { minimum le risque d'utiliser un produit contrefait }(36,37,54,60)\end{array}$ \\
\hline
\end{tabular}

Certains antipaludéens sont contre-indiqués pour le traitement du paludisme (auto-traitement ou autre) :

- méfloquine $(61,62)$;

- sulfadoxine/pyriméthamine (Fansidar) (63);

- méfloquine/Fansidar (62);

- halofantrine (39);

- chloroquine/Fansidar (59).

\section{Prophylaxie finale}

$P$. vivax et $P$. ovale peuvent persister dans le foie et causer des rechutes jusqu'à cinq ans après le départ d'une région impaludée. Le traitement anti-rechute à la primaquine réduit le risque de rechute en agissant contre les stades hépatiques de $P$. vivax et $P$. ovale. Le traitement anti-rechute à la primaquine est généralement prescrit pendant ou après les deux dernières semaines de la chimioprophylaxie aux personnes qui étaient dans des régions impaludées (la plupart des régions impaludées du monde, à l'exception de Haïti et de la République dominicaine) $(26,38,39,64)$. Le traitement anti-rechute à la primaquine est contre-indiqué chez les personnes présentant un déficit en G-6-PD, les femmes enceintes et les femmes qui allaitent si le nourrisson présente un déficit en G-6-PD.

\section{Voyageurs souffrant de comorbidités}

Des interactions entre le paludisme et d'autres problèmes médicaux sous-jacents peuvent entraîner une plus grande sensibilité au paludisme et une gravité accrue de ce dernier, ou encore des complications des problèmes médicaux sous-jacents en question. Certains problèmes médicaux sous-jacents peuvent être aggravés par un ou plusieurs antipaludéens, voire en proscrire totalement l'usage. 
Entreprendre systématiquement une vérification de l'interaction médicamenteuse pour éviter tout potentiel d'interactions médicamenteuses indésirables, à moins que l'innocuité des médicaments du voyageur soit connue avec l'antipaludéen proposé.

\section{Hôtes immunodéprimés}

Les voyageurs immunodéprimés devraient soigneusement respecter les mesures de protection individuelle et la chimioprophylaxie.

$\mathrm{VIH} /$ sida

Des interactions importantes et complexes ont lieu entre le virus de l'immunodéficience humaine (VIH) et $P$. falciparum. Évaluer les interactions médicamenteuses et tenir compte du risque de chevauchement des profils d'effets indésirables (65). Le CCMTMV recommande de consulter un spécialiste en médecine des voyages/tropicale ou en maladies infectieuses et le spécialiste du VIH du voyageur (40).

\section{Asplénie}

L'asplénie augmente le risque, l'ampleur et la durée de la parasitémie, même chez les personnes partiellement immunisées dans les pays impaludés (41), et le risque de paludisme grave et mortel chez des voyageurs souffrant d'asplénie (66). Il faut recommander, en plus des mesures prophylactiques, un auto-traitement de réserve, si le sujet doit se rendre dans des régions isolées ou dans des régions où l'accès aux soins médicaux est limité. Puisque la fièvre peut être due au paludisme ou à une infection bactérienne, il faut prescrire un traitement antibactérien de réserve (67).

\section{Autres maladies}

Une liste des autres maladies et de leurs répercussions sur le choix de la chimioprophylaxie antipaludéenne est résumée dans le Tableau 4.

Tableau 4 : Autres maladies influant sur le choix de la chimioprophylaxie antipaludéenne

\begin{tabular}{|c|c|}
\hline Maladie & Répercussions sur le choix de la chimioprophylaxie antipaludéenne \\
\hline $\begin{array}{l}\text { Coagulation } \\
\text { anormale }\end{array}$ & $\begin{array}{l}\text { La méfloquine, la doxycycline et le proguanil peuvent potentialiser la warfarine }(42-45,68) \text {. } \\
\text { Il faut effectuer un essai de traitement médicamenteux plusieurs semaines avant le voyage et des } \\
\text { tests en série du Rapport international normalisé (RIN) pour permettre un rajustement de la } \\
\text { dose d'anticoagulant avant et après le voyage. }\end{array}$ \\
\hline Troubles convulsifs & $\begin{array}{l}\text { Comme la chloroquine et la méfloquine peuvent exacerber certains troubles convulsifs, il faut } \\
\text { prescrire d'autres agents. Rien n'indique que les convulsions fébriles chez les enfants soient une } \\
\text { contre-indication de ces médicaments. } \\
\text { Une utilisation simultanée des médicaments anticonvulsifs qui induisent des enzymes } \\
\text { microsomales hépatiques (p. ex. barbituriques, phénytoïne, carbamazépine) peut réduire les } \\
\text { taux sériques et la demi-vie de la doxycycline, et peut nécessiter un rajustement de la dose (45). }\end{array}$ \\
\hline
\end{tabular}




\begin{tabular}{|c|c|}
\hline Myasthénie grave & $\begin{array}{l}\text { Les infections palustres peuvent exacerber la myasthénie grave. Il faut renforcer la prévention } \\
\text { optimale par l'observance de la chimioprophylaxie et des mesures de protection individuelle. } \\
\text { Éviter la chloroquine, la méfloquine et la doxycycline, car elles ont été associées à l'aggravation } \\
\text { des symptômes de myasthénie. On peut envisager la doxycycline chez les patients stables, en } \\
\text { particulier ceux qui ne présentent que des symptômes ophtalmologiques, bien que le CCMTMV } \\
\text { recommande un essai du traitement avant le voyage. Un essai avant le voyage du traitement par } \\
\text { atovaquone/proguanil est recommandé, car il a été signalé que la monothérapie au proguanil } \\
\text { aggravait les symptômes de myasthénie (9). } \\
\text { La primaquine n'a pas été associée à des symptômes de myasthénie et peut représenter une } \\
\text { option pour la prophylaxie contre l'infection à P. falciparum (après avoir éliminé tout déficit en } \\
\text { G-6-PD) chez les voyageurs souffrant de myasthénie qui ne peuvent tolérer la doxycycline et } \\
\text { l'atovaquone/proguanil. }\end{array}$ \\
\hline $\begin{array}{l}\text { Troubles } \\
\text { psychiatriques }\end{array}$ & $\begin{array}{l}\text { Évaluer les antécédents de dépression, de trouble anxieux généralisé ou de psychose avant de } \\
\text { prescrire de la méfloquine }(46,70) \text {. } \\
\text { Des effets neuropsychiatriques indésirables liés à la dose sont bien connus avec la méfloquine et, } \\
\text { à un degré moindre, avec la chloroquine }(71,72) \text {. }\end{array}$ \\
\hline $\begin{array}{l}\text { Troubles hépatiques } \\
\text { ou rénaux }\end{array}$ & $\begin{array}{l}\text { Des troubles hépatiques ou rénaux modérés ou graves peuvent modifier les concentrations } \\
\text { sériques d'antipaludéens*. Au besoin, il faut consulter un spécialiste en médecine des } \\
\text { voyages/tropicale. } \\
\text { Une insuffisance rénale grave (clairance de la créatinine }<30 \mathrm{~mL} / \mathrm{min} \text { ) est une contre-indication } \\
\text { à l'utilisation de l'atovaquone/proguanil. }\end{array}$ \\
\hline Psoriasis & Éviter la chloroquine, car elle peut déclencher des éruptions aiguës de psoriasis $(73,74)$. \\
\hline $\begin{array}{l}\text { Déficit en glucose-6- } \\
\text { phosphate } \\
\text { déshydrogénase (G- } \\
6 \text {-PD) }\end{array}$ & $\begin{array}{l}\text { La primaquine est associée à un risque d'hémolyse potentiellement mortel. Bien que le déficit en } \\
\text { G-6-PD soit une préoccupation des fabricants de chloroquine, les spécialistes ne considèrent pas } \\
\text { cela comme une contre-indication, car une hémolyse importante est peu probable avec les doses } \\
\text { prophylactiques. }\end{array}$ \\
\hline Porphyrie & $\begin{array}{l}\text { Mis à part l'atovaquone/proguanil (75), tous les agents chimioprophylactiques de première } \\
\text { intention contre le paludisme peuvent être porphyrinogènes. À utiliser avec prudence. }\end{array}$ \\
\hline
\end{tabular}

*Consulter le Tableau 5.4.3 des Recommandations canadiennes pour la prévention et le traitement du paludisme (malaria) chez les voyageurs internationaux, pour connaître les éléments à prendre en compte en matière d'antipaludéens pour les personnes souffrant de troubles hépatiques ou rénaux.

\section{Conclusion}

Certains groupes de voyageurs ont besoin de renseignements supplémentaires pour la prévention et la prise en charge du paludisme. En outre, ils doivent reconnaître l'importance de l'observance des recommandations en matière de chimioprophylaxie et des mesures de protection individuelle. Le traitement varie selon l'espèce de plasmodies en cause, la gravité du paludisme et la région où la maladie a été contractée, et selon les interactions potentielles entre les médicaments utilisés pour le traitement des maladies chroniques et le traitement antipaludéen recommandé.

\section{Remerciements}

La présente déclaration a été préparée par Boggild A., Brophy J., Charlebois P., Crockett M., Geduld J., Ghesquiere W., McDonald P., Plourde P., Teitelbaum P., Tepper M., Schofield S. et McCarthy A.

Le CCMTMV tient à remercier Joanna Odrowaz et Elspeth Payne pour leur contribution à l'élaboration des sommaires et Manisha Kulkarni pour sa contribution à la déclaration. 
Membres du CCMTMV : Boggild A., Brophy J., Bui Y. G., Crockett M., Ghesquiere W., Greenaway C., Henteleff A., Libman M., Teitelbaum P. et McCarthy A. (présidente)

Représentants chargés de la liaison : Hui C. (Société canadienne de pédiatrie) et Gershman M. (Centers for Disease Control and Prevention [É.-U.])

Membres d'office : Marion D. (Centre des services de santé des Forces canadiennes, ministère de la Défense nationale), McDonald P. (Division des médicaments anti-infectieux, Santé Canada), Schofield S. (Direction de la protection de la santé de la Force, ministère de la Défense nationale) et Tepper M. (Direction de la protection de la santé de la Force, ministère de la Défense nationale)

Membre émérite : Jeanes C. W. L.

\section{Conflit d'intérêts}

Il n'y a aucun conflit d'intérêts à déclarer.

\section{Financement}

Ce travail a été appuyé par l'Agence de la santé publique du Canada.

\section{Références}

(1) McCarthy AE, Plourde P, Kuhn S, Bodie M. Parenteral quinine for severe malaria: Five year surveillance data from the Canadian Malaria Network. 10th Conference of the International Society of Travel Medicine 2007;Abstract No. FC02.01.

(2) Comité consultative de la medicine tropicale et de la medicine des voyages. Recommandations canadiennes pour la prévention et le traitement du paludisme chez les voyageurs internationaux (malaria).

http://publications.gc.ca/site/fra/463471/publication.html

(3) Boggild A, Brophy J, Charlebois P, Crockett M, Geduld J, Ghesquiere W, et al. Résumé des recommandations sur la prevention du paludisme du CCMTMV. RMTC 2014;40(7):118.Boggild A., Brophy J., Charlebois P., Crockett M., Geduld J., Ghesquiere W.et al

(4) Boggild A, Brophy J, Charlebois P, Crockett M, Geduld J, Ghesquiere W, et al. Summary of recommendations for the diagnosis and treatment of malaria by the Committee to Advise on Tropical Medicine and Travel (CATMAT). Can Comm Dis Rep 2014;40(7):131.Boggild A., Brophy J., Charlebois P., Crockett M., Geduld J., Ghesquiere W. et al.

(5) Maitland K. M, K. Pathophysiology of severe malaria in children. Acta Trop 2004;90(2):131.

(6) American Academy of Pediatrics editor. Red Book: 2009 Report of the Committee on Infectious Diseases. 28th ed. Illinois: American Academy of Pediatrics; 2009.

(7) Fradin MS, Day JF. Comparative Efficacy of Insect Repellents against Mosquito Bites. N Engl J Med 2002 07/04; 2014/02;347(1):13-18.

(8) Committee to Advise on Tropical Medical and Travel. Statement on personal protective measures to prevent arthropod bites. Canadian Communicable Disease Report 2012;38(ASC-3):1-18. 
(9) Centers for Disease Control and Prevention. Information for health care providers: preventing malaria in infants and children. 2006; http://wwwnc.cdc.gov/travel.

(10) Camus D, Djossou F, Schilthuis HJ, Hogh B, Dutoit E. Atovaquone-proganil versus chloroquine-proguanil for malaria prophylaxis in nonimmune pediatric travelers: results of an international, randomized, open-label study. Clin Infect Dis 2004;38(12):1716-1723.

(11) Pang LW, Limsomwong N, Boudreau EF, Singharaj P. Doxycycline prophylaxis for falciparum malaria. Lancet 1987;329(8543):1161-1164.

(12) Overbosch D, Schilthuis H, Bienzle U, Behrens RH, Kain KC, Clarke PD, et al. Atovaquone-Proguanil versus Mefloquine for Malaria Prophylaxis in Nonimmune Travelers: Results from a Randomized, Double-Blind Study. Clinical Infectious Diseases 2001 October 01;33(7):1015-1021.

(13) Weiss W, Oloo A, Johnson A, Koech D, Hoffman S. Daily primaquine is effective for prophylaxis against falciparum malaria in Kenya: comparison with mefloquine, doxycycline, and chloroquine plus proguanil. J Infectious Dis 1995;171(6):1569-75.

(14) Martindale editor. The Complete Drug Reference. 32nd ed. London: Pharmaceutical Press; 2008.

(15) Shulman CE, Dorman EK. Importance and prevention of malaria in pregnancy. Trans R Soc Trop Med Hyg 2003 0;97(1):30-35.

(16) McGready R, Hamilton K, Simpson JA, Cho J, Luxemberger C, Edwards R, et al. Safety of the insect repellent N,Ndietyl-M-toluide (DEET) in pregnancy. Am J Trop Med Hyg 2001;65(4):285-289.

(17) Nosten F, Vincenti M, Simpson J, Yei P, Thwai KL, Vries Ad, et al. The Effects of Mefloquine Treatment in Pregnancy. Clin Infect Dis 1999 Apr.;28(4):808-815.

(18) Balocco R, Bonati M. Melfoquine prophylaxis against malaria for female travelers of childbearing age. Lancet 1992;340(8814):309-310.

(19) Steketee RW, Wirima JJ, Slutsker L, Roberts JM, Khoromana CO, Heymann DL, et al. Malaria parasite infection during pregnancy and at delivery in mother, placenta, and newborn: efficacy of chloroquine and mefloquine in rural Malawi. The American Journal of Tropical Medicine and Hygiene 1996 July 01;55(1 Suppl):24-32.

(20) Stauffer WM, Kamat D, Magill AJ. Traveling with Infants and Children. Part IV: Insect Avoidance and Malaria Prevention. Journal of Travel Medicine 2003;10(4):225-240.

(21) Na-Bangchang K, Manyando C, Ruengweerayut R, Kioy D, Mulenga M, Miller G, et al. The pharmacokinetics and pharmacodynamics of atovaquone and proguanil for the treatment of uncomplicated falciparum malaria in thirdtrimester pregnant women. Eur J Clin Pharmacol 2005 Sep;61(8):573-582.

(22) Hogh B, Clarke PD, Camus D, Nothdurft HD, Overbosch D, Gunther M, et al. Atovaquone-proguanil versus chloroquine-proguanil for malaria prophylaxis in non-immune travellers: a randomised, double-blind study. Malarone International Study Team. Lancet 2000 Dec 2;356(9245):1888-1894.

(23) American Academy of Pediatrics Committee on Drugs. Transfer of drugs and other chemicals into human milk. Pediatrics 2001 Sep;108(3):776-789. 
(24) Mali S, Kachur SP, Arguin PM, Division of Parasitic Diseases and Malaria, Center for Global Health, Centers for Disease Control and Prevention (CDC). Malaria surveillance--United States, 2010. MMWR Surveill Summ 2012 Mar 2;61(2):1-17.

(25) N, Stauffer B, Boulware DR, Walker PF, Keystone JS. Travel medicine considerations for North American immigrants visiting friends and relatives. JAMA 2004 Jun 16;291(23):2856-2864 (seulement disponible en Anglais).

(26) Chen LH, Wilson ME, Schlagenhauf P. Prevention of malaria in long-term travelers. JAMA 2006 Nov 8;296(18):2234-2244.

(27) Berg J, Visser LG. Expatriate chemoprophylaxis use and compliance: past, present and future from an occupational health perspective. J Travel Med 2007 Sep-Oct;14(5):357-358.

(28) Schlagenhauf P, Petersen E. Malaria chemoprophylaxis: strategies for risk groups. Clin Microbiol Rev 2008 Jul;21(3):466-472.

(29) Korhonen C, Peterson K, Bruder C, Jung P. Self-reported adverse events associated with antimalarial chemoprophylaxis in peace corps volunteers. Am J Prev Med 2007 Sep;33(3):194-199.

(30) Schlagenhauf P, Tschopp A, Johnson R, Nothdurft HD, Beck B, Schwartz E, et al. Tolerability of malaria chemoprophylaxis in non-immune travellers to sub-Saharan Africa: multicentre, randomised, double blind, four arm study. BMJ 2003 Nov 8;327(7423):1078.

(31) Knobloch J. Long-term malaria prophylaxis for travelers. J Travel Med 2004 Nov-Dec;11(6):374-378.

(32) Hamer DH, Ruffing R, Callahan MV, Lyons SH, Abdullah AS. Knowledge and use of measures to reduce health risks by corporate expatriate employees in western Ghana. J Travel Med 2008 Jul-Aug;15(4):237-242.

(33) Dahlgren AL, Deroo L, Avril J, Bise G, Loutan L. Health risks and risk-taking behaviors among International Committee of the Red Cross (ICRC) expatriates returning from humanitarian missions. J Travel Med 2009 NovDec;16(6):382-390.

(34) Funk M, Schlagenhauf P, Tschopp A, Steffen R. MalaQuick versus ParaSight F as a diagnostic aid in travellers' malaria. Trans R Soc Trop Med Hyg 1999 May-Jun;93(3):268-272.

(35) Dondorp AM, Newton PN, Mayxay M, Van Damme W, Smithuis FM, Yeung S, et al. Fake antimalarials in Southeast Asia are a major impediment to malaria control: multinational cross-sectional survey on the prevalence of fake antimalarials. Trop Med Int Health 2004 Dec;9(12):1241-1246.

(36) Newton P, Proux S, Green M, Smithuis F, Rozendaal J, Prakongpan S, et al. Fake artesunate in southeast Asia. Lancet 2001 Jun 16;357(9272):1948-1950.

(37) Cockburn R, Newton PN, Agyarko EK, Akunyili D, White NJ. The global threat of counterfeit drugs: why industry and governments must communicate the dangers. PLoS Med 2005 Apr;2(4):e100.

(38) Kotwal RS, Wenzel RB, Sterling RA, Porter WD, Jordan NN, Petruccelli BP. An outbreak of malaria in US Army Rangers returning from Afghanistan. JAMA 2005 Jan 12;293(2):212-216.

(39) World Health Organization. Guidelines for the treatment of malaria, Second edition. 2010. 
(40) Centers for Disease Control and Prevention (CDC). CDC Health Information for International Travel $2012<\mathrm{br} />$. New York: Oxford University Press; 2012.

(41) Bach O, Baier M, Pullwitt A, Fosiko N, Chagaluka G, Kalima M, et al. Falciparum malaria after splenectomy: a prospective controlled study of 33 previously splenectomized Malawian adults. Trans R Soc Trop Med Hyg 2005 Nov;99(11):861-867.

(42) Westfall LK, Mintzer DL, Wiser TH. Potentiation of warfarin by tetracycline. Am J Hosp Pharm 1980 Dec;37(12):1620, 1625.

(43) Loefler I. Mefloquine and anticoagulant interaction. J Travel Med 2003 May-Jun;10(3):194-195.

(44) Armstrong G, Bed MF, Scahill S. Warfarin potentiated by proguanil. BMJ 1991 Sep 28;303(6805):789.

(45) Doxycycline: Cautions (Drug Interactions). 2007;Report No.:10-4-0007.

(46) Schlagenhauf P. Mefloquine for malaria chemoprophylaxis 1992-1998: a review. J Travel Med 1999 Jun;6(2):122133.

(47) Lobel HO, Varma JK, Miani M, Green M, Todd GD, Grady K, et al. Monitoring for mefloquine-resistant Plasmodium falciparum in Africa: implications for travelers' health. Am J Trop Med Hyg 1998 Jul;59(1):129-132.

(48) Lobel HO, Bernard KW, Williams SL, Hightower AW, Patchen LC, Campbell CC. Effectiveness and tolerance of long-term malaria prophylaxis with mefloquine. Need for a better dosing regimen. JAMA 1991 Jan 16;265(3):361-364.

(49) Lobel HO, Miani M, Eng T, Bernard KW, Hightower AW, Campbell CC. Long-term malaria prophylaxis with weekly mefloquine. Lancet 1993 Apr 3;341(8849):848-851.

(50) Ohrt C, Richie T, Widjaja H, Shanks G, Fitriadi J, Fryauff D, et al. Mefloquine compared with doxycycline for the prophylaxis of malaria in Indonesian soldiers. A randomized, double-blind, placebo-controlled trial. Ann Intern Med 1997;126(12):963-72.

(51) Kain KC, MacPherson DW, Kelton T, Keystone JS, Mendelson J, MacLean JD. Malaria deaths in visitors to Canada and in Canadian travellers: a case series. Canadian Medical Association Journal 2001 March 06;164(5):654-659.

(52) Marx A, Pewsner D, Egger M, Nuesch R, Bucher HC, Genton B, et al. Meta-analysis: accuracy of rapid tests for malaria in travelers returning from endemic areas. Ann Intern Med 2005 May 17;142(10):836-846.

(53) Jelinek T, Amsler L, Grobusch MP, Nothdurft HD. Self-use of rapid tests for malaria diagnosis by tourists. Lancet 1999 Nov 6;354(9190):1609.

(54) Schlagenhauf P, Steffen R. Stand-by treatment of malaria in travellers: a review. J Trop Med Hyg 1994 Jun;97(3):151-160.

(55) Schlagenhauf P, Steffen R, Tschopp A, Van Damme P, Mittelholzer ML, Leuenberger H, et al. Behavioural aspects of travellers in their use of malaria presumptive treatment. Bull World Health Organ 1995;73(2):215-221.

(56) Schlagenhauf P, Petersen E. Standby emergency treatment of malaria in travelers: experience to date and new developments. Expert Rev Anti Infect Ther 2012 May;10(5):537-546. 
(57) Swales CA, Chiodini PL, Bannister BA, Health Protection Agency Advisory Committee on Malaria Prevention in UK Travellers. New guidelines on malaria prevention: A summary. J Infect 2007 Feb;54(2):107-110.

(58) Quach C, Kain K, MacPherson D, Mendelson J, MacLean J. Malaria deaths in Canadian travellers. Can Commun Dis Rep 1999 Mar 15;25(6):50-53.

(59) Chen LH, Wilson ME, Schlagenhauf P. Controversies and misconceptions in malaria chemoprophylaxis for travelers. JAMA 2007 May 23;297(20):2251-2263.

(60) Nothdurft HD, Jelinek T, Pechel SM, Hess F, Maiwald H, Marschang A, et al. Stand-by treatment of suspected malaria in travellers. Trop Med Parasitol 1995 Sep;46(3):161-163.

(61) Weinke T, Trautmann M, Held T, Weber G, Eichenlaub D, Fleischer K, et al. Neuropsychiatric side effects after the use of mefloquine. Am J Trop Med Hyg 1991 Jul;45(1):86-91.

(62) Luxemburger C, Price RN, Nosten F, Ter Kuile FO, Chongsuphajaisiddhi T, White NJ. Mefloquine in infants and young children. Ann Trop Paediatr 1996 Dec;16(4):281-286.

(63) Roll Back Malaria Department WHOU. World Malaria Report. 2005.

(64) Hill DR, Baird JK, Parise ME, Lewis LS, Ryan ET, Magill AJ. Primaquine: report from CDC expert meeting on malaria chemoprophylaxis I. Am J Trop Med Hyg 2006 Sep;75(3):402-415.

(65) Khoo S, Back D, Winstanley P. The potential for interactions between antimalarial and antiretroviral drugs. AIDS 2005 Jul 1;19(10):995-1005.

(66) Demar M, Legrand E, Hommel D, Esterre P, Carme B. Plasmodium falciparum malaria in splenectomized patients: two case reports in French Guiana and a literature review. Am J Trop Med Hyg 2004 Sep;71(3):290-293.

(67) Baker H. The influence of chloroquine and related drugs on psoriasis and keratoderma. Br J Dermatol 1966;78:161.

(68) Touze JE, Heno P, Fourcade L, Deharo JC, Thomas G, Bohan S, et al. The effects of antimalarial drugs on ventricular repolarization. Am J Trop Med Hyg 2002 Jul;67(1):54-60.

(69) Fischer PR, Walker E. Myasthenia and malaria medicines. J Travel Med 2002 Sep-Oct;9(5):267-268.

(70) Schlagenhauf P, Adamcova M, Regep L, Schaerer MT, Rhein HG. The position of mefloquine as a 21st century malaria chemoprophylaxis. Malar J 2010 Dec 9;9:357-2875-9-357.

(71) Barrett PJ, Emmins PD, Clarke PD, Bradley DJ. Comparison of adverse events associated with use of mefloquine and combination of chloroquine and proguanil as antimalarial prophylaxis: postal and telephone survey of travellers. BMJ 1996 Aug 31;313(7056):525-528.

(72) Akhtar S, Mukherjee S. Chloroquine induced mania. Int J Psychiatry Med 1993;23(4):349-356.

(73) Kuflik EG. Effect of antimalarial drugs on psoriasis. Cutis 1980 Aug;26(2):153-155.

(74) Fry L, Baker BS. Triggering psoriasis: the role of infections and medications. Clin Dermatol 2007 NovDec;25(6):606-615. 
(75) The Drug Database for Acute Porphyria. http://www.drugs-porphyria.org/monograph.php?id=4079. 